\title{
Adaptive Control of Space Robot Despinning Tumbling Target Using Flexible Brushes
}

\author{
Shengxin Sun $\mathbb{D}^{1},{ }^{1}$ Cheng Wei $\mathbb{D}^{1},{ }^{1}$ Zhuoran Huang $\mathbb{D}^{1}{ }^{1}$ Hao Wu $\mathbb{D},{ }^{2}$ Haibo Zhang $\mathbb{D}^{3}{ }^{3}$ \\ Jianchun Lu iD, ${ }^{4}$ and Yan Du iD ${ }^{5}$ \\ ${ }^{1}$ Harbin Institute of Technology, Harbin 150001, China \\ ${ }^{2}$ KUKA Robotics Guangdong Co., Ltd., Foshan 528311, China \\ ${ }^{3}$ Beijing Institute of Control Engineering, Beijing 100190, China \\ ${ }^{4}$ China Academy of Space Technology, Beijing 100000, China \\ ${ }^{5}$ Information Engineering University, Zhengzhou 450001, China
}

Correspondence should be addressed to Shengxin Sun; 16b918086@stu.hit.edu.cn

Received 12 June 2021; Revised 9 July 2021; Accepted 28 July 2021; Published 26 September 2021

Academic Editor: Shunan Wu

Copyright ( 2021 Shengxin Sun et al. This is an open access article distributed under the Creative Commons Attribution License, which permits unrestricted use, distribution, and reproduction in any medium, provided the original work is properly cited.

\begin{abstract}
A flexible brush mechanism is designed and mounted at the end of a seven-degree-of-freedom robotic arm to despin a tumbling target. The dynamics model of the flexible brush is established using the absolute nodal coordinate method (ANCF), and its contact collision with the solar wing of the tumbling target is analysed. The $H_{\infty}$ optimal control is proposed for a sevendegree-of-freedom robotic arm during despinning of a tumbling target while ensuring the global robustness and stability. Simulations verify that the despinning strategy can successfully eliminate the rotation speed and is feasible and effective.
\end{abstract}

\section{Introduction}

With the development of space technology, the number of human space satellites has gradually increased and the resulting space debris removal has become a key topic in the space industry [1]. In order to control the growth of the space debris population and eliminate its threat to spaceflight activities, active space debris removal techniques have become a current research hotspot [2]. Capture strategy for non-cooperative targets tumbling at high speed, applying resistance and reducing their angular speed of rotation to make capture less difficult [3]. In terms of contact despinning, Huang et al. $[4,5]$ proposed a method for attitude control of noncooperative targets based on a tether terminal, which stabilizes the attitude of the tumbling target by controlling the tether tension and damping force attached to it; Daneshjou and Alibakhshi [6] proposed a spring damper buffer device which is accomplished by contact collision dur- ing nozzle docking in the despinning process; Nishida and Kawamoto [7] designed a despinning device with a flexible brush as the end-effector, which uses the elastic contact force between the brush and the target for despinning. There are also space debris removal systems such as drag-increasing devices, which accelerate the target deconvolution process by increasing the surface-to-mass ratio of the target, thus increasing the air drag; methods include spraying foam on space debris $[8,9]$ and installing airbags for space debris [10].

For the stability of the deconvolution mechanism during the deconvolution process, the fast response and stability of the robotic arm are achieved by using sliding mode control. In terms of the sliding mode control, Oliveira et al. [11] proposed an adaptive sliding mode method based on the concept of extended equivalent control to deal with disturbances at unknown boundaries in nonlinear systems to avoid overestimation of controller gains and loss of sliding 
motion. Kawamura et al. [12] proposed a sliding mode control design based on a disturbance observer using Lyapunov's stability theorem method, which reduces the gain of the switching term in the sliding mode controller and effectively eliminates jitter.

Veysi et al. [13] designed a fuzzy sliding mode controller for controlling the position of an end-effector in a space task. This controller uses a new heuristic algorithm, the adaptive improved BAT algorithm, to determine the coefficients of the fuzzy sliding-mode controller and verify its performance with a two-degree-of-freedom robotic arm. Mobayen et al. [14] investigated a second-order fast terminal sliding mode control technique based on linear matrix inequalities for a class of tracking problems with unmatched nonlinear uncertainty, which showed significant improvements in both control performance and tracking performance. Karimi et al. $[15,16]$ solved the control problem for a class of uncertain nonlinear systems using a generalized adaptive control. Sun and Hou [17] studied the control problem of a flexible linkage robotic arm with uncertainty and proposed a sliding mode control method with both a neural network and a disturbance observer for adaptive design. The update laws of the neural network and disturbance observer were designed on this basis, and the stability of the closed-loop system was proved by Lyapunov analysis. Mobayen et al. [18] designed an adaptive super-torsional global nonlinear sliding mode control rate for an N-linked robot, which ensured the elimination of the arrival phase and the presence of the sliding mode on the right side of the surface, while using the adaptive control law to eliminate external disturbances. Yang et al. [19, 20] designed an adaptive neural network for sliding mode control of flexible manipulators. Cao et al. $[21,22]$ use a robust fixed-time attitude stabilization control with spacecraft with actuator uncertainty. Zhang et al. [23-25], with regard to the issues of stability for hidden semi-Markov jump systems, designed a stabilizing controller dependent on both the observed mode, and the elapsed time it is constructed. In the sense of $\sigma$-error mean square stability ( $\sigma$-MSS), numerically testable criteria on the basis of semi-Markov kernel (SMK) and emission probability of HS-MJLSs are obtained.

As a brand new research topic, despinning is still in the theoretical stage at home and abroad. This paper designs a robust optimal controller based on linear quadratic performance indicators for a seven-degree-of-freedom robotic arm with uncertainty and external disturbances. The essence of the control is to minimise the parametrization of the error transfer function under the condition that the disturbance is bounded, considering the maximum disturbance that the system may withstand. At the same time, the optimal control makes the system robustly optimal for a specified performance index. By analysing Lyapunov stability, it can be demonstrated that in the case of bounded disturbances, the robust state feedback control term in the controller can effectively compensate for the uncertainties in the system and external disturbances, allowing the robot arm to accurately track the desired trajectory, i.e., the closed-loop system achieves asymptotic stability, while the quadratic performance index is optimal.

\section{Despin Dynamics}

This paper designs a space robot with a flexible reduction brush at the end to deconvolve a tumbling target in space. The deconfliction mechanism is flexible and has soft contact characteristics for noncooperative targets in a free tumbling state, which can improve the safety of deconfliction. The model is shown in Figure 1.

Definition of the coordinate system and symbols: coordinate system $\Sigma_{\mathrm{I}}$ is the inertial coordinate system, coordinate system $\Sigma_{\mathrm{b}}$ is the base body coordinate system, coordinate system $\Sigma_{\mathrm{t}}$ is the target body coordinate system, $q=\left[q_{1}, q_{2}\right.$, $\left.q_{3}, q_{4}, q_{5}, q_{6}, q_{7}\right]$ are the robot arm joint variables, $q_{b}=\left[q_{b 1}\right.$, $\left.q_{b 2}, q_{b 3}\right]$ is the attitude Euler angle of the base body, $C_{i}$ is the mass center of the $K$ th rod of the robot arm, $J_{i}$ is the joint connecting the $i-1$ and $i$ rods, ${ }^{I} r_{i}$ is the position of the mass center of the $i$ rod of the robot arm in the inertial coordinate system, ${ }^{I} r_{0}$ is the position vector of the mass center of the base spacecraft in the inertial coordinate system, ${ }^{I} r_{\mathrm{g}}$ is the position vector of the mass center of the space robot system, ${ }^{I} \omega_{i}$ is the angular velocity of the $i$ rod of the arm, ${ }^{I} \omega_{0}$ is the angular velocity of the base, ${ }^{I} \omega_{t}$ is the angular velocity of the target, and $m_{i}$ is the mass of the $i$ rod of the space robot. The top left corner I indicates the representation of the vector in the inertial coordinate system.

2.1. Modeling of Rope Dynamics Based on ANCF Cable-Beam Units. The flexible reduction brush at the end of the gyroscopic robot is modelled using rope dynamics based on the Absolute Nodal Coordinate Formulation (ANCF) cablebeam element.

2.1.1. Kinetic Energy of Cable Element. Since the shape function of the cable element is constant, the velocity vector at any point on the $j$ cable element can be written as

$$
{ }^{j} \dot{r}=S^{j} \dot{q}_{\mathrm{c}}
$$

where $r$ is the global position vector at any point, $S$ is the shape function, and $q_{\mathrm{c}}$ is the generalized coordinates.

Using equation (1), the kinetic energy of the flexible cable element can be written as

${ }^{j} T=\frac{1}{2} \int_{0}^{L} \rho \int_{A}^{j} \dot{q}_{\mathrm{c}}^{\mathrm{T}} S^{\mathrm{T}} S^{\mathrm{j}} \dot{q}_{\mathrm{c}} \mathrm{d} A \mathrm{~d} x=\frac{1}{2} \dot{q}_{\mathrm{c}}^{\mathrm{T}}\left(\int_{0}^{L} \rho\left(A S^{\mathrm{T}} S\right) \mathrm{d} x\right){ }^{j} \dot{q}_{\mathrm{c}}=\frac{1}{2}{ }^{j} \dot{q}_{\mathrm{c}}^{\mathrm{T}^{\mathrm{j}}} M^{\mathrm{j}} \dot{q}_{\mathrm{c}}$,

where $\rho$ and $A$ are the cable element density and crosssectional area, respectively; $L$ is the cable element length; and $M=\int_{0}^{L} \rho\left(A S^{\mathrm{T}} S\right) \mathrm{d} x$ represents the constant mass matrix of the cable element $[19,20]$.

2.1.2. Internal Energy of the Element. Using the BernoulliEuler beam equation, the flexible deceleration brush cable is subjected to preload and the element positive stress is

$$
\sigma=E \varepsilon+\sigma_{1}
$$




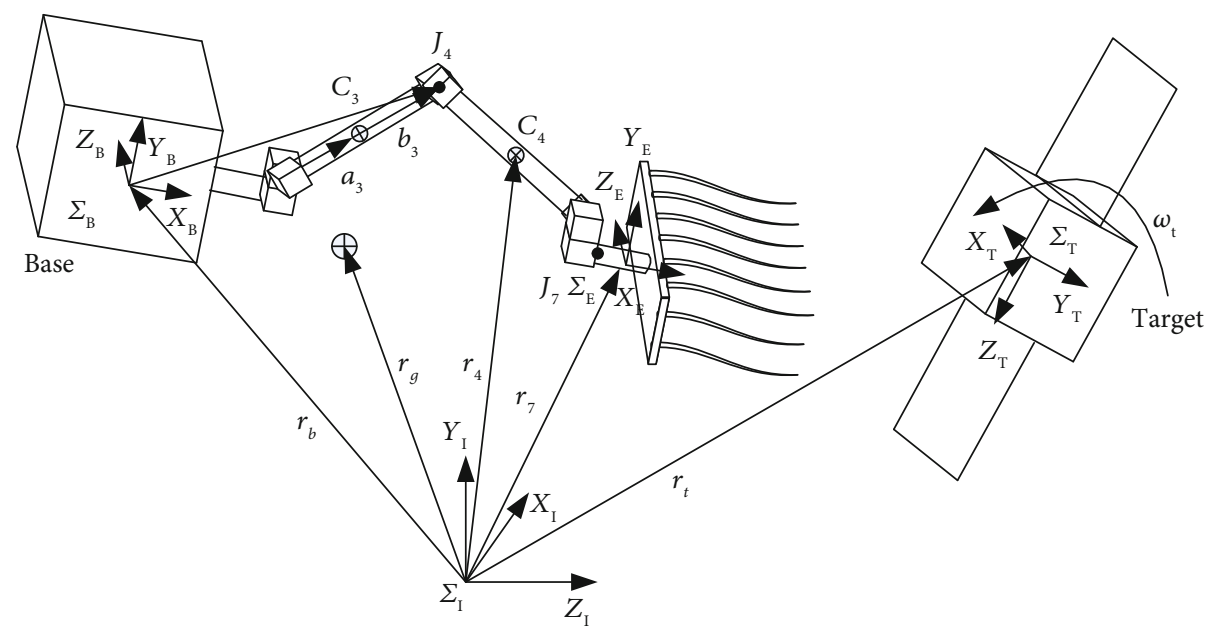

Figure 1: A model of seven-degree-of-freedom manipulator.

where $\sigma_{1}$ is the preload force, $E$ is the modulus of elasticity, and $\varepsilon$ is the axial strain. Then, the internal energy of the flexible cable element is expressed as

$$
U=\int_{V}\left(\frac{1}{2} E \varepsilon^{2}+\sigma_{1} \varepsilon\right) \mathrm{d} V=\mathrm{AL} \int_{0}^{1}\left(\frac{1}{2} E \varepsilon^{2}+\sigma_{1} \varepsilon\right) \mathrm{d} x,
$$

where $V$ is the volume of the flexible cable unit.

2.1.3. Kinetic Equations. The total kinetic energy and total strain energy of the flexible cable system are

$$
\left\{\begin{array}{l}
T=\sum_{j=1}^{3 k} T=\frac{1}{2} \dot{q}_{\mathrm{c}}^{\mathrm{T}} M \dot{q}_{\mathrm{c}}, \\
U=\sum_{j=1}^{3 k} U=\sum_{j=1}^{3 k} \mathrm{AL} \int_{0}^{1}\left(\frac{1}{2} E^{j} \varepsilon^{2}+{ }^{j} \sigma_{1}{ }^{j} \varepsilon\right) \mathrm{d} x .
\end{array}\right.
$$

The kinetic equation for the flexible cable system is

$$
\left\{\begin{array}{l}
\frac{\mathrm{d}}{\mathrm{d} t}\left(\frac{\partial T}{\partial \dot{q}_{\mathrm{c}}}\right)^{\mathrm{T}}-\left(\frac{\partial T}{\partial q_{\mathrm{c}}}\right)^{\mathrm{T}}+\left(\frac{\partial U}{\partial q_{\mathrm{c}}}\right)^{\mathrm{T}}+\left(\frac{\partial D}{\partial q_{\mathrm{c}}}\right)^{\mathrm{T}} \lambda=Q_{\mathrm{e}} \\
D\left(q_{\mathrm{c}}, t\right)=0
\end{array}\right.
$$

where $D$ is the constraint equation, $\lambda$ is the Rasch multiplier corresponding to the constraint equation, $Q_{e}$ is the generalized force vector, and $q_{\mathrm{c}}$ and $\lambda$ are both unknown quantities.

2.2. Contact Collision Dynamics Modelling. During the derotation process, the contact collision occurs mainly between the flexible reduction brush and the sail of the tumbling target. The contact collision between the flexible reduction brush and the target is modelled based on a nonlinear spring damping model. According to Hertz collision theory, the contact collision is represented as

$$
\left\{\begin{array}{l}
F=\left(F_{\mathrm{K}}+F_{\mathrm{C}}\right) B(\delta)=\left(K \delta^{n}+C \dot{\delta}\right) B(\delta), \\
B(\delta)= \begin{cases}0 & \delta \geq 0, \\
1 & \delta<0 .\end{cases}
\end{array}\right.
$$

$F_{\mathrm{K}}$ is the normal collision force, $F_{\mathrm{C}}$ is the normal damping force, $K$ is the equivalent contact stiffness, $C$ is the equivalent contact damping factor, $\delta$ is the normal penetration depth, $\dot{\delta}$ is the normal penetration velocity, $n$ is the exponent $(n \geq 1) . B(\delta)$ is a logistic function that determines whether contact is made based on the normal penetration $\delta$.

2.3. Modelling the Dynamics of Free-Floating Space Robots. The equations for the dynamics of a free-floating space robot are

$$
\left[\begin{array}{cc}
H_{\mathrm{b}} & H_{\mathrm{bm}} \\
H_{\mathrm{bm}}^{\mathrm{T}} & H_{\mathrm{m}}
\end{array}\right]\left[\begin{array}{c}
\ddot{x}_{\mathrm{b}} \\
\ddot{q}_{\mathrm{m}}
\end{array}\right]+\left[\begin{array}{c}
c_{\mathrm{b}} \\
c_{\mathrm{m}}
\end{array}\right]=\left[\begin{array}{c}
F_{\mathrm{b}} \\
\tau
\end{array}\right]+\left[\begin{array}{c}
J_{\mathrm{b}}^{\mathrm{T}} \\
J_{\mathrm{m}}^{\mathrm{T}}
\end{array}\right] F_{\mathrm{e}},
$$

where $H_{\mathrm{b}}$ is the base inertia matrix; $H_{\mathrm{m}}$ is the arm inertia matrix; $H_{\mathrm{bm}}$ is the space robot coupling inertia matrix; $x_{\mathrm{b}}$ is the base position; $q_{\mathrm{m}}$ is the arm joint angle; $c_{\mathrm{b}}$ is the nonlinear term of the base; $c_{\mathrm{m}}$ is the nonlinear term of the arm; $F_{\mathrm{b}}$ and $F_{\mathrm{e}}$ are the forces acting on the base and the end, respectively; $\tau$ is the arm joint moment; $J_{\mathrm{b}}$ is the end base Jacobi matrix; and $J_{\mathrm{m}}$ is the end joint Jacobi matrix.

2.4. Robust Control Dynamics Modelling. Dynamics modelling has been completed for space robots:

$$
H\left(q_{\mathrm{tog}}\right) \ddot{q}_{\mathrm{tog}}+C\left(q_{\mathrm{tog}}, \dot{q}_{\mathrm{tog}}\right) \dot{q}_{\mathrm{tog}}=\tau,
$$

where $H\left(q_{\mathrm{tog}}\right)$ is the symmetric positive definite inertia matrix; $C\left(q_{\text {tog }}, \dot{q}_{\text {tog }}\right) \dot{q}_{\text {tog }}$ is the nonlinear coupling term: $C($ 
TABLE 1: Kinetic parameters.

\begin{tabular}{|c|c|c|c|c|c|c|c|c|}
\hline Parameter name & Matrix & Joint 1 & Joint 2 & Joint 3 & Joint 4 & Joint 5 & Joint 6 & Joint 7 \\
\hline Length $a(\mathrm{~m})$ & - & 0 & 0 & 1 & 1 & 0 & 0 & 0 \\
\hline Angle $\alpha\left(^{\circ}\right)$ & - & 90 & 90 & 0 & 0 & 90 & 90 & 0 \\
\hline Joint distance $d(\mathrm{~m})$ & - & 0 & 0.1 & 0 & 0 & 0.3 & 0.1 & 0 \\
\hline Joint angle $\theta\left(^{\circ}\right)$ & - & $\theta 1$ & $\theta 2$ & $\theta 3$ & $\theta 4$ & $\theta 5$ & $\theta 6$ & $\theta 7$ \\
\hline Mass $m(\mathrm{~kg})$ & 2811 & 2.8 & 2.8 & 25.39 & 25.39 & 2.8 & 2.8 & 10.6 \\
\hline $\mathrm{Ix}\left(\mathrm{kg} \cdot \mathrm{m}^{2}\right)$ & 473 & 0.005 & 0.005 & 2.76 & 2.76 & 0.005 & 0.005 & 1.33 \\
\hline Iy $\left(\mathrm{kg} \cdot \mathrm{m}^{2}\right)$ & 473 & 0.005 & 0.005 & 2.76 & 2.76 & 0.005 & 0.005 & 1 \\
\hline $\mathrm{Iz}\left(\mathrm{kg} \cdot \mathrm{m}^{2}\right)$ & 467 & 0.005 & 0.005 & 0.03 & 0.03 & 0.005 & 0.005 & 1.5 \\
\hline
\end{tabular}

TABLE 2: Spin target parameters.

\begin{tabular}{lcccccc}
\hline $\begin{array}{l}m \\
(\mathrm{~kg})\end{array}$ & $\begin{array}{c}\text { Substrate } \\
\text { volume } \\
\left(\mathrm{m}^{3}\right)\end{array}$ & $\begin{array}{c}\text { Sail area } \\
\left(\mathrm{m}^{2}\right)\end{array}$ & $\begin{array}{c}\text { Rolling } \\
\text { speed } \\
\left(\mathrm{rad} \cdot \mathrm{s}^{-1}\right)\end{array}$ & $\begin{array}{c}\mathrm{Ix} \\
\left(\mathrm{kg} \cdot \mathrm{m}^{2}\right)\end{array}$ & $\begin{array}{c}\mathrm{Iy} \\
\left(\mathrm{kg} \cdot \mathrm{m}^{2}\right)\end{array}$ & $\begin{array}{c}\mathrm{Iz} \\
\left(\mathrm{kg} \cdot \mathrm{m}^{2}\right)\end{array}$ \\
\hline 2000 & $1 \times 1 \times 1$ & $1 \times 5.113$ & 1 & 200 & 300 & 200 \\
\hline
\end{tabular}

$\left.q_{\text {tog }}, \dot{q}_{\text {tog }}\right) \dot{q}_{\text {tog }}=\dot{H} \dot{q}_{\text {tog }}-\left(\partial / \partial q_{\text {tog }}\right)\left((1 / 2) \dot{q}_{\text {tog }}^{\mathrm{T}} H \dot{q}_{\text {tog }}\right)$; and $\tau$ is the forces and moments acting on the base and robotic arm joints.

The space ablative robot is in the complex microgravity environment of space and contains a flexible reduction brush at the end, so considering the uncertainties of external disturbances an friction and parameter errors, equation (9) can be expressed as

$$
\begin{gathered}
H\left(q_{\mathrm{tog}}\right) \ddot{q}_{\mathrm{tog}}+C\left(q_{\mathrm{tog}}, \dot{q}_{\mathrm{tog}}\right) \dot{q}_{\mathrm{tog}}=\tau, \\
H\left(q_{\mathrm{tog}}\right)=H_{0}\left(q_{\mathrm{tog}}\right)+\Delta H\left(q_{\mathrm{tog}}\right), \\
C\left(q_{\mathrm{tog}}, \dot{q}_{\mathrm{tog}}\right)=C_{0}\left(q_{\mathrm{tog}}, \dot{q}_{\mathrm{tog}}\right)+\Delta C\left(q_{\mathrm{tog}}, \dot{q}_{\mathrm{tog}}\right),
\end{gathered}
$$

where $H_{0}\left(q_{\text {tog }}\right)$ and $C_{0}\left(q_{\text {tog }}, \dot{q}_{\text {tog }}\right)$ are the knowable nominal matrix and $\Delta H\left(q_{\text {tog }}\right)$ and $\Delta C\left(q_{\text {tog }}, \dot{q}_{\text {tog }}\right)$ are the unknowable nominal matrix.

$H_{0}\left(q_{\text {tog }}\right) \ddot{q}_{\text {tog }}+C_{0}\left(q_{\text {tog }}, \dot{q}_{\text {tog }}\right) \dot{q}_{\text {tog }}=\tau-\Delta H\left(q_{\text {tog }}\right) \ddot{q}_{\text {tog }}-\Delta C\left(q_{\text {tog }}, \dot{q}_{\text {tog }}\right) \dot{q}_{\text {tog }}$.

Dynamic compensation is as follows:

$$
\begin{gathered}
\tau=H_{0}\left(q_{\mathrm{tog}}\right) u+C_{0}\left(q_{\mathrm{tog}}, \dot{q}_{\mathrm{tog}}\right) \dot{q}_{\mathrm{tog}}, \\
\ddot{q}_{\mathrm{tog}}=u-H_{0}^{-1} \Delta H \ddot{q}_{\mathrm{tog}}-H_{0}^{-1} \Delta C \dot{q}_{\mathrm{tog}} .
\end{gathered}
$$

$u$ is the control input vector, defining external disturbances $\tau_{d}$ :

$$
\delta\left(q_{\mathrm{tog}}, \dot{q}_{\mathrm{tog}}, \ddot{q}_{\mathrm{tog}}\right)=-\left(\Delta H \ddot{q}_{\mathrm{tog}}+\Delta C \dot{q}_{\mathrm{tog}}-\tau_{d}\right)
$$

In order to make the space robot end track the desired trajectory with time variation, the state tracking error is defined as $e \in R^{13}$ :

$$
e=\left[\begin{array}{l}
\dot{q}_{\text {tog }}-\dot{q}_{\text {tog }}^{d} \\
q_{\text {tog }}-q_{\text {tog }}^{d}
\end{array}\right]=\left[\begin{array}{c}
\dot{\tilde{q}}_{\text {tog }} \\
\tilde{q}_{\text {tog }}
\end{array}\right],
$$

where $q_{\text {tog }}^{d}$ is the expected arm joint angles and $\dot{q}_{\text {tog }}^{d}$ is the desired angular velocity of the joint of the manipulator.

The trajectory tracking error state equation of the space robot can be obtained as follows:

$$
\dot{e}=A\left(q_{\mathrm{tog}}, \dot{q}_{\mathrm{tog}}\right) e+B u+B w .
$$

Each parameter is specifically defined as

$$
\begin{gathered}
A\left(q_{\mathrm{tog}}, \dot{q}_{\mathrm{tog}}\right)=\left[\begin{array}{cc}
-H_{0}^{-1} C_{0} & 0 \\
I_{13} & 0
\end{array}\right], \\
B=\left[I_{13} 0\right]^{\mathrm{T}}, \\
w=-H_{0}^{-1} \delta\left(q_{\mathrm{tog}}, \dot{q}_{\mathrm{tog}}, \ddot{q}_{\mathrm{tog}}\right), \\
u=H_{0}^{-1}\left(\tau-H_{0} \ddot{q}_{\mathrm{tog}}^{d}-C_{0} \dot{q}_{\mathrm{tog}}^{d}\right) .
\end{gathered}
$$

Therefore, the force and moment exerted on the space robot can be solved as

$$
\tau=H_{0}\left(\ddot{q}_{\mathrm{tog}}^{d}+u\right)+C_{0} \dot{q}_{\mathrm{tog}}^{d}
$$

To track the desired trajectory and reach the desired point at the end, the design aid equation is as follows:

$$
z=D e=\left[\begin{array}{cc}
D_{11} & D_{12} \\
0 & I
\end{array}\right]\left[\begin{array}{l}
\dot{\tilde{q}}_{\text {tog }} \\
\tilde{q}_{\text {tog }}
\end{array}\right],
$$

where $D_{11}$ and $D_{12}$ are constant matrices.

Putting formula (16) to formula (10),

$$
\dot{e}=A_{N} e+B_{N} u+B_{N} w .
$$



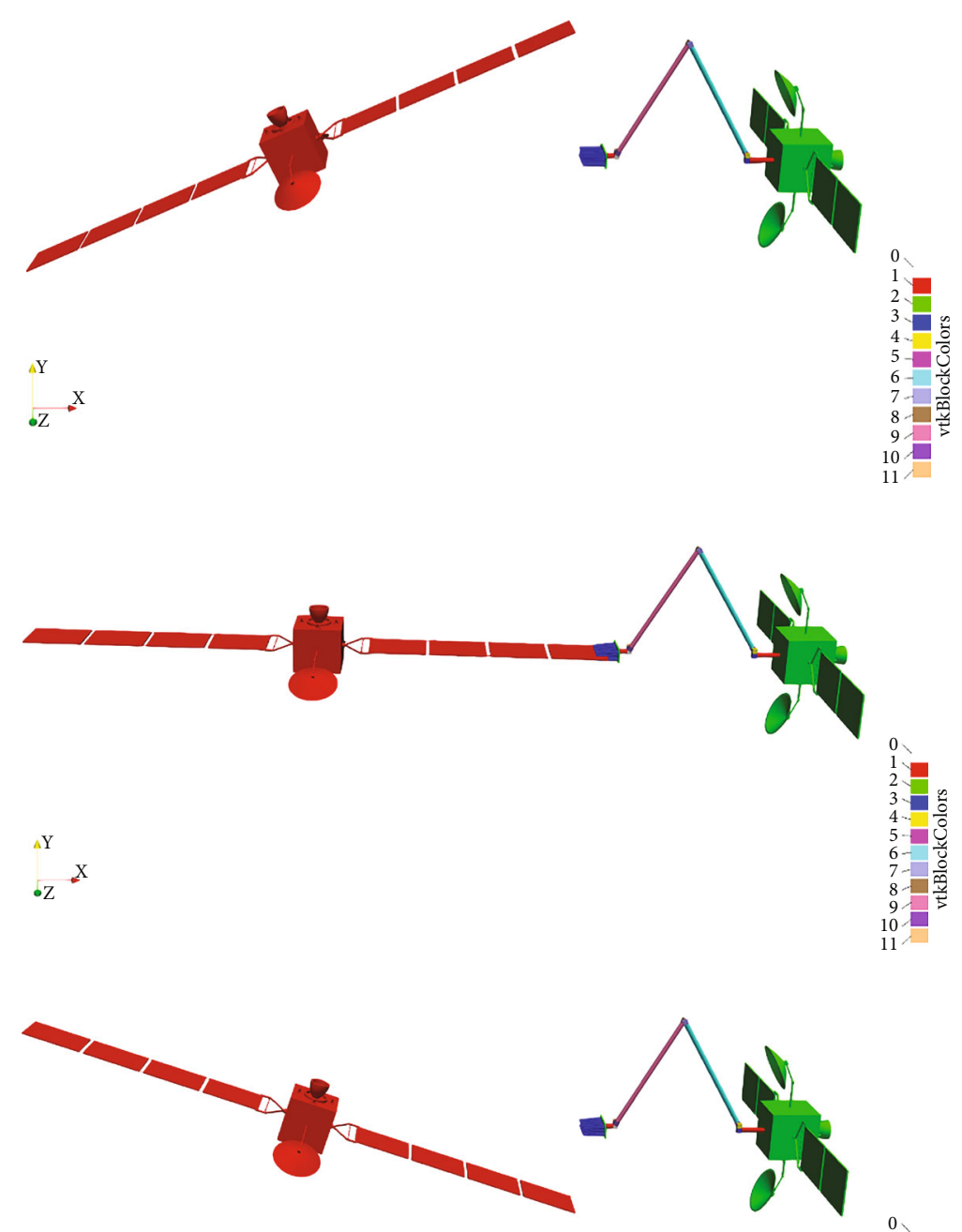

$\stackrel{\triangle \mathrm{Y}}{\mathrm{A}} \mathrm{X}$

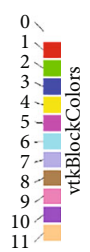

Figure 2: Spin satellite derotation effect.

In formula (17),

$$
\begin{gathered}
A_{N}=D^{-1}\left[\begin{array}{cc}
-H_{0}^{-1} C_{0} & 0 \\
D_{11}^{-1} & -D_{11}^{-1} D_{12}
\end{array}\right] D \\
B_{N}=D^{-1}\left[\begin{array}{c}
H_{0}^{-1} \\
0
\end{array}\right] \\
D_{1}=\left[D_{11} \quad D_{12}\right] \\
u=H_{0} D_{1} \dot{e}+C_{0} D_{1} e, \\
w=H_{0} D_{11} H_{0}^{-1} \delta\left(q_{\mathrm{tog}}, \dot{q}_{\mathrm{tog}}, \ddot{q}_{\mathrm{tog}}\right) .
\end{gathered}
$$

Then, the control force and moment of the space robot can be expressed as

$$
\begin{aligned}
& \tau=H_{0} \ddot{q}_{\mathrm{tog}}+C_{0} \dot{q}_{\mathrm{tog}}^{d}, \\
& \ddot{q}_{\text {tog }}=\ddot{q}_{\text {tog }}^{d}+\left(-D_{11}^{-1} H_{0}^{-1} C_{0} D_{11}-D_{11}^{-1} D_{12}\right) \dot{e}-D_{11}^{-1} H_{0}^{-1} C_{0} D_{12} e+D_{11}^{-1} H_{0}^{-1} u
\end{aligned}
$$

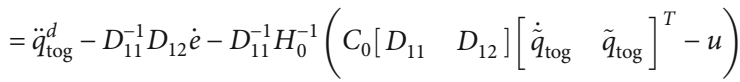

$$
\begin{aligned}
& =\ddot{q}_{\mathrm{tog}}^{d}-D_{11}^{-1} D_{12} \dot{\tilde{q}}_{\mathrm{tog}}-D_{11}^{-1} H_{0}^{-1}\left(C_{0} D_{1} \dot{e}-u\right) \text {. }
\end{aligned}
$$

Designing the control law $u=-K e(t)$, the external disturbance $w$ in the system is reduced. $\gamma$ is any positive real number.

$$
J=\min _{u \in L_{2}} \max _{0 \neq w \in L_{2}} \frac{\int_{0}^{\infty}\left((1 / 2) e^{\mathrm{T}} Q e+(1 / 2) u^{\mathrm{T}} R u\right) d t}{\int_{0}^{\infty}\left((1 / 2) w^{\mathrm{T}} w\right) d t} \leq \gamma^{2} .
$$




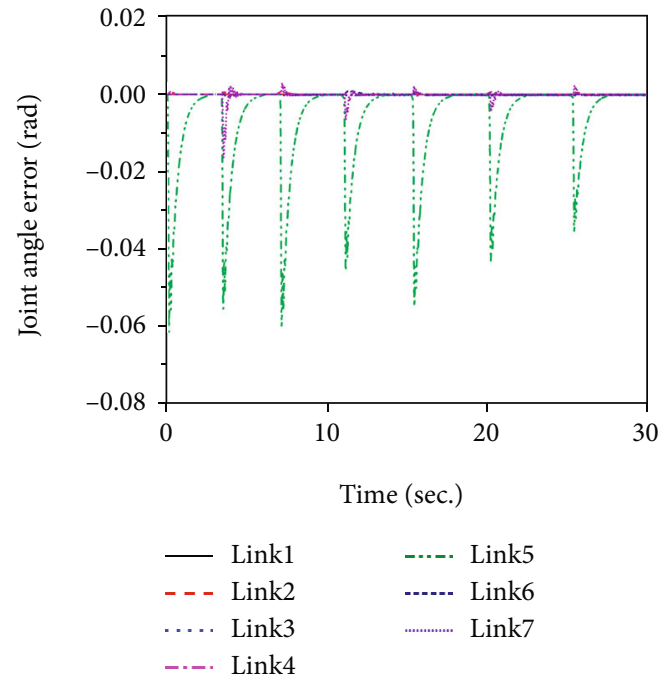

Figure 3: Joint angle error.

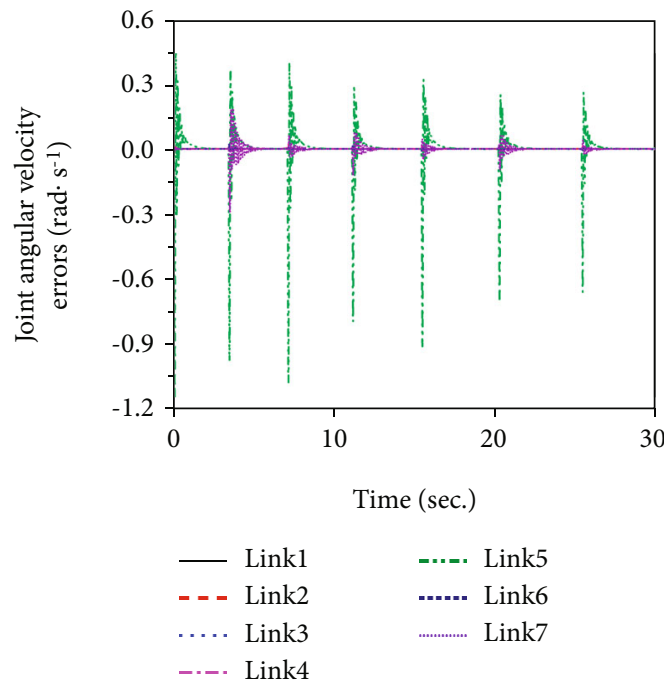

Figure 4: Joint angular velocity error.

Designing the Lyapunov function $V(x)=(1 / 2) x^{\mathrm{T}} P x$, the Riccati equation is solved as follows:

$$
\dot{P}+P A_{N}+A_{N} P+P B_{N}\left(R^{-1}-\frac{1}{\gamma^{2}} I\right) B_{N} P+Q=0 .
$$

By the skew symmetric matrix $C_{0}-(1 / 2) M_{0}$, we design function $P$ as follows:

$$
P=D^{T}\left[\begin{array}{cc}
M_{0} & 0 \\
0 & N
\end{array}\right] D
$$

where $N$ is a positive definite symmetric constant matrix.

Putting formula (27) in formula (26),

$$
\left[\begin{array}{cc}
0 & N \\
N & 0
\end{array}\right]-D^{\mathrm{T}} B_{N}\left(R^{-1}-\frac{1}{\gamma^{2}} I\right) B_{N}^{\mathrm{T}} D+Q=0 .
$$

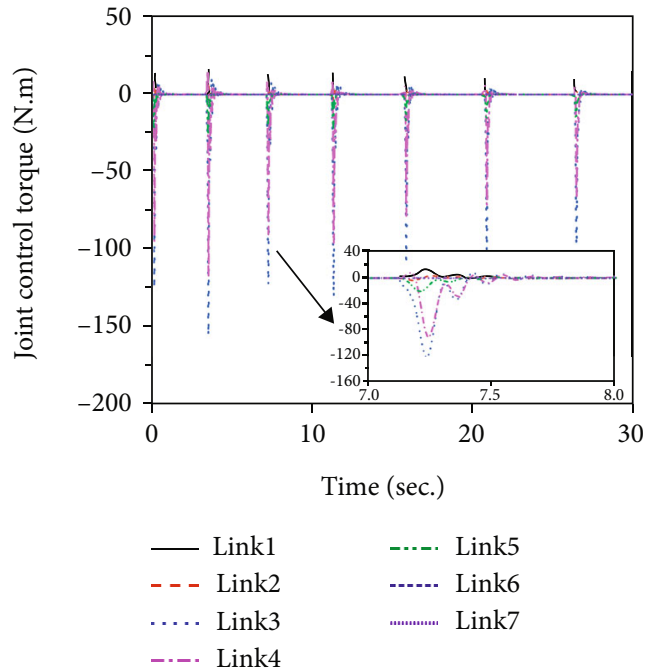

Figure 5: Joint control torque.

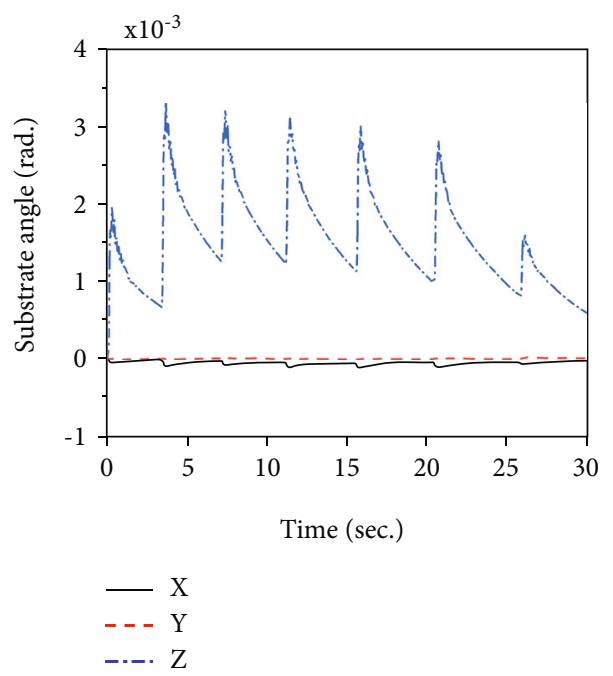

FIgURE 6: Substrate angle.

By Cholesky decomposition, we get $R_{1}$ :

$$
R_{1}^{\mathrm{T}} R_{1}=\left(R^{-1}-\frac{1}{\gamma^{2}} I\right)^{-1}
$$

At the same time,

$$
\begin{gathered}
Q=\left[\begin{array}{cc}
Q_{1}^{\mathrm{T}} Q_{1} & Q_{12} \\
Q_{12}^{\mathrm{T}} & Q_{2}^{\mathrm{T}} Q_{2}
\end{array}\right], \\
D=\left[\begin{array}{cc}
R_{1}^{\mathrm{T}} Q_{1} & R_{1}^{\mathrm{T}} Q_{2} \\
0 & I
\end{array}\right], \\
N=\frac{1}{2}\left(Q_{1}^{T} Q_{2}+Q_{2}^{T} Q_{1}\right)-\frac{1}{2}\left(Q_{21}^{T}+Q_{12}\right) .
\end{gathered}
$$

So, $H_{\infty}$ the state feedback control rate of the robust 


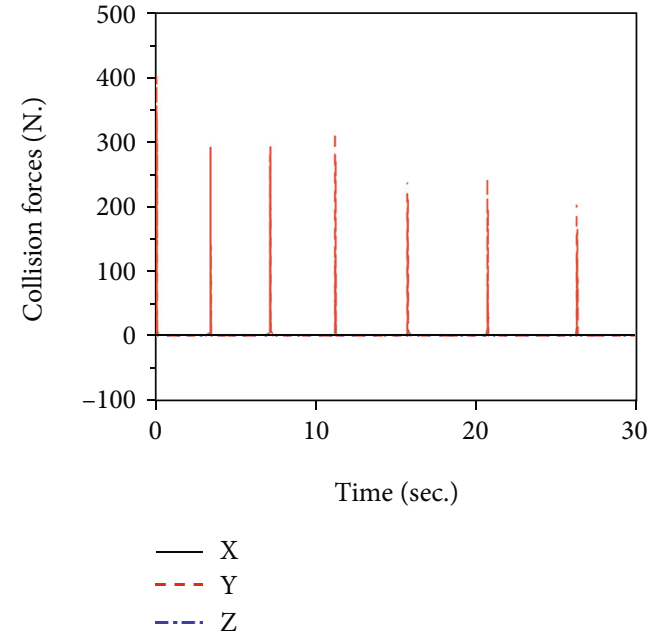

Figure 7: Collision forces.

optimal controller is

$$
u=-R^{-1} B^{\mathrm{T}} D e .
$$

Remark 1. In [26], the authors mainly considered the stabilization problem of the flexible deceleration brush detumbling mechanism attached to a space robot arm, where an optimal adaptive control scheme and a backstepping control strategy with sliding mode differentiator are proposed. Different from [26], in this paper, we mainly discuss the stabilization issue of the manipulator under complex environmental conditions and we show that the developed despinning strategy can successfully eliminate the rotation speed.

\section{Spin Target Decontrol Study}

The redundant robotic arm has seven degrees of freedom, and the floating base has six degrees of freedom. The $\mathrm{DH}$ method was used to build the space robot structure with the dynamics parameters shown in Table 1. The parameters of the designed space spin target are shown in Table 2. The target is initially rotated around the axis with an angular velocity of the actual effect as shown in Figure 2.

A robust optimal controller is used to simulate the deconvolution of a spatial target in a spin state. The target mass center and the mass center of the space robot are located in the plane of deconvolution, and the initial position of the end flexible brush is set in this plane. Therefore, for the spin target deconvolution, once the deconvolution plane is determined, it is only necessary to maintain the initial joint angle of the robot arm to ensure that the end flexible reduction brush reaches the deconvolution position before the next deconvolution.

The arm's joint angle error and joint angular velocity error are shown in Figures 3 and 4, respectively. The joint angle error occurs during the contact collision process, where the instantaneous force causes a large deviation in the joint angle of the robot arm, which is then controlled

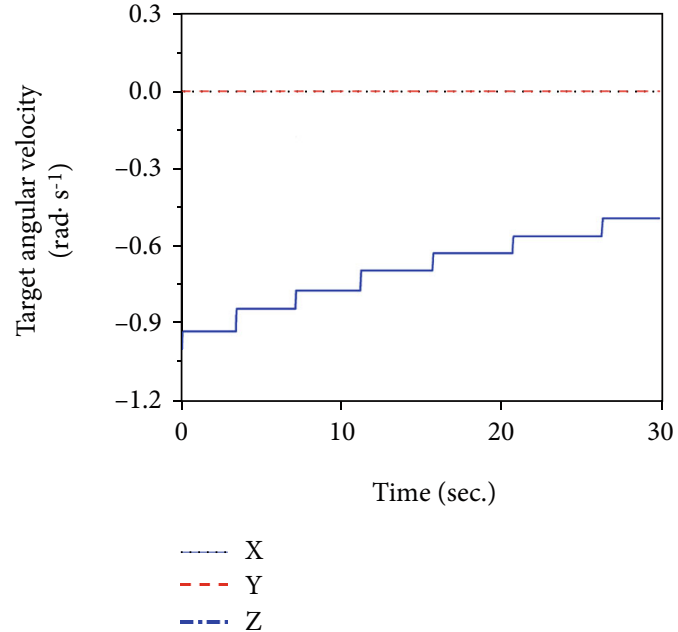

FIgURE 8: Target spin angular velocity.

by the controller to recover the initial position. The magnitude of the joint angle error is in the range of $0.06 \mathrm{rad}$, and the convergence time is approximately $3 \mathrm{~s}$. The joint angle error due to the contact collision gradually decreases as the deconvolution progresses. The joint angular velocity error also decreases in magnitude.

The robot arm control torque is shown in Figure 5: the initial solving interval time is about $3.5 \mathrm{~s}$ and the initial joint control torque is in the range of $150 \mathrm{~N}$. As the solving proceeds and the solving interval time increases, the solving torque gradually decreases. The simulation time for the spin target in this section is $30 \mathrm{~s}$, in which a total of 7 spin cycles are performed. Later in the $30 \mathrm{~s}$ spin process, i.e., during the 6th and 7th spins, the spin interval has grown to $5.6 \mathrm{~s}$ and the joint control torque drops to within 100 N. Extracting the third deconvolution process for analysis shows that the initial joint moments are similarly large during the 7 to $8 \mathrm{~s}$ period when the contact collision causes a sudden increase in the robot arm joint error. At the end of the collision deconvolution, the control torque converges to zero within $0.8 \mathrm{~s}$, completing the stable control of the system.

The substrate angle is shown in Figure 6. The motion of the robotic arm and the collision interference at the end affect the base angle of the space robot. The error accuracy of the base angle is $0.23^{\circ} / \mathrm{s}$.

During the spin-up process, the spin target is subjected to contact collision forces, as shown in Figure 7 . The collision forces occur mainly in the direction perpendicular to the spin plane, which in this case is the $y$-axis direction. The magnitude of the collision force is in the range of $400 \mathrm{~N}$ and decays with time. The collision force is applied at the deconfliction position at the edge of the target sail, with the point of action and the length of the sail at the mass center of the target acting as the deconfliction arm, both forming a despinning moment for despinning.

The change of the angular velocity of the spin target is shown in Figure 8. The angular velocity of the spin target decreases from $1 \mathrm{rad} / \mathrm{s}$ to $0.4944 \mathrm{rad} / \mathrm{s}$ within $30 \mathrm{~s}$ of deconvolution time, and the deconvolution effect is $49.44 \%$. 
Therefore, it can be proven that the deconvolution efficiency and $H_{\infty}$ optimal controller designed in this paper can achieve the desired deconvolution effect. Due to the effectiveness and stability of the controller, the target has a good despinning effect and no large chapter motion in the other two axes. However, the spatial environment is complex and uncertain, and there is a high probability that the spin target will be converted into a chapter motion under the condition of unknown disturbance.

\section{Conclusions}

A $H_{\infty}$ optimal controller is designed to address the external disturbances and uncertainties in the space robot deconvolution process. Using the Lyapunov stability theory, it is demonstrated that under the condition of bounded disturbances, the robust state feedback control term can be designed to compensate for uncertainties and unknown disturbances to achieve the purpose of deconvolution, thus ensuring the global asymptotic stability of the closed-loop system. It is also verified that the controller is able to demonstrate high accuracy and good convergence in both unknown disturbances and uncertain environments. The ability of the flexible reduction brush end designed in this paper to perform the deconvolution task also demonstrates the accuracy of the controller and the effectiveness of the deconvolution strategy. Simulations using the $H_{\infty}$ optimal controller for autonomous deconvolution control show that the $H_{\infty}$ optimal controller has high accuracy and good convergence.

\section{Data Availability}

The datasets generated and analysed during the current study are available from the corresponding author on reasonable request. The authors declare that the data supporting the findings of this study are available within the article.

\section{Conflicts of Interest}

The authors declare that they have no conflicts of interest.

\section{Acknowledgments}

The study was supported by the Science and Technology on Space Intelligent Control Laboratory (6142208180402).

\section{References}

[1] Y. LU, X. LIU, and Y. ZHOU, "Review of detumbling technologies for active removal of uncooperative targets," Acta Aeronautica et Astronautica Sinica, vol. 39, no. 1, pp. 38-50, 2018.

[2] X. ZHOU, The simulation analysis of active debris removal of multiple targets in a single task, Beijing: The University of Chinese Academy of Sciences, in Chinese, 2017.

[3] Y. GENG, W. LU, and X. CHEN, "Attitude synchronization control of on-orbit servicing spacecraft with respect to outof-control target," Journal of Harbin Institute of Technology, vol. 44, no. 1, pp. 1-6, 2012.

[4] P. Huang, M. Wang, Z. Meng, F. Zhang, Z. Liu, and H. Chang, "Reconfigurable spacecraft attitude takeover control in post- capture of target by space manipulators," Journal of the Franklin Institute, vol. 353, no. 9, pp. 1985-2008, 2016.

[5] P. Huang, F. Zhang, Z. Meng, and Z. Liu, "Adaptive control for space debris removal with uncertain kinematics, dynamics and states," Acta Astronautica, vol. 128, pp. 416-430, 2016.

[6] K. Daneshjou and R. Alibakhshi, "Multibody dynamical modeling for spacecraft docking process with spring-damper buffering device: a new validation approach," Advances in Space Research, vol. 61, no. 1, pp. 497-512, 2018.

[7] S.-I. Nishida and S. Kawamoto, "Strategy for capturing of a tumbling space debris," Acta Astronautica, vol. 68, no. 1-2, pp. 113-120, 2011.

[8] A. NATARAJAN and H. SCHAUB, "Hybrid control of orbit normal and along-track two-craft Coulomb tethers," Aerospace Science and Technology, vol. 13, no. 4-5, pp. 183-191, 2009.

[9] P. PERGOLA and A. RUGGIERO, "Expanding foam application for active space debris removal systems," in Proceedings of 62nd International Astronautical Congress (IAC11), 2011.

[10] L. LIN, "Status and removal of space debris," Spacecraft Engineering, vol. 21, no. 3, 2012.

[11] T. R. Oliveira, J. P. V. S. Cunha, and H. Liu, "Adaptive sliding mode control based on the extended equivalent control concept for disturbances with unknown bounds," Studies in Systems, Decision and Control, vol. 115, pp. 149-163, 2018.

[12] A. Kawamura, H. Ito, and K. Sakamoto, "Chattering reduction of disturbance observer based sliding mode control," Transactions on industry applications, vol. 30, no. 2, pp. 456-461, 1994.

[13] M. Veysi, M. R. Soltanpour, and M. H. Khooban, "A novel selfadaptive modified bat fuzzy sliding mode control of robot manipulator in presence of uncertainties in task space," Robotica, vol. 33, no. 10, pp. 2045-2064, 2015.

[14] S. Mobayen, D. Baleanu, and F. Tchier, "Second-order fast terminal sliding mode control design based on LMI for a class of non-linear uncertain systems and its application to chaotic systems," Journal of Vibration and Control, vol. 23, pp. 2912-2925, 2016.

[15] Z. Liu, H. R. Karimi, and J. Yu, "Passivity-based robust sliding mode synthesis for uncertain delayed stochastic systems via state observer," Automatica, vol. 111, p. 108596, 2020.

[16] J. Zhai and H. R. Karimi, "Universal adaptive control for uncertain nonlinear systems via output feedback," Information Sciences, vol. 500, pp. 140-155, 2019.

[17] H. B. Sun and L. L. Hou, "Composite anti-disturbance attitude and vibration control for flexible spacecraft," IET Control Theory \& Applications, vol. 11, no. 14, pp. 2383-2390, 2017.

[18] S. Mobayen, F. Tchier, and L. Ragoub, "Design of an adaptive tracker for n-link rigid robotic manipulators based on supertwisting global nonlinear sliding mode control," International Journal of Systems Science, vol. 48, no. 9, pp. 1990-2002, 2017.

[19] H. Yang and M. Tan, "Sliding mode control for flexible-link manipulators based on adaptive neural networks," International Journal of Automation and Computing, vol. 15, no. 2, pp. 239-248, 2018.

[20] H. Yang and J. Liu, “An adaptive RBF neural network control method for a class of nonlinear systems," IEEE/CAA Journal of Automatica Sinica, vol. 5, no. 2, pp. 457-462, 2018.

[21] L. Cao, B. Xiao, and M. Golestani, "Robust fixed-time attitude stabilization control of flexible spacecraft with actuator uncertainty," Nonlinear Dynamics, vol. 100, no. 3, pp. 2505-2519, 2020. 
[22] B. Xiao, L. Cao, and S. Y. Xu, "Robust tracking control of robot manipulators with actuator faults and joint velocity measurement uncertainty," IEEE/ASME Transactions on Mechatronics, vol. 25, no. 3, pp. 1354-1365, 2020.

[23] L. X. Zhang, B. Cai, and Y. Shi, "Stabilization of hidden semiMarkov jump systems: emission probability approach," Automatica, vol. 101, pp. 87-95, 2019.

[24] B. Cai, L. X. Zhang, and Y. Shi, "Observed-mode-dependent state estimation of hidden semi-Markov jump linear systems," IEEE Transactions on Automatic Control, vol. 65, no. 1, pp. 442-449, 2019.

[25] L. X. Zhang, B. Cai, T. Tan, and Y. Shi, "Stabilization of nonhomogeneous hidden semi-Markov jump systems with limited sojourn-time information," Automatica, vol. 101, no. 7-8, pp. 87-95, 2020.

[26] S. Sun, Y. Zhao, and H. Wu, "Optimal adaptive control and backstepping control method with sliding mode differentiator," Complexity, vol. 2021, 15 pages, 2021. 